\title{
Evolution of “51 Pegasus b-like" planets
}

\author{
T. Guillot ${ }^{1}$ and A. P. Showman ${ }^{2}$ \\ 1 Observatoire de la Côte d'Azur, Laboratoire Cassini, CNRS UMR 6529, 06304 Nice Cedex 4, France \\ 2 University of Arizona, Department of Planetary Sciences and Lunar and Planetary Lab, Tucson, AZ 85721 USA \\ e-mail: showman@lpl.arizona.edu
}

Rceived 23 March 2001 / Accepted 7 November 2001

\begin{abstract}
About one-quarter of the extrasolar giant planets discovered so far have orbital distances smaller than 0.1 AU. These "51 Peg b-like" planets can now be directly characterized, as shown by the planet transiting in front the star HD 209458. We review the processes that affect their evolution. We apply our work to the case of HD 209458b, whose radius has been recently measured. We argue that its radius can be reproduced only when the deep atmosphere is assumed to be unrealistically hot. When using more realistic atmospheric temperatures, an energy source appears to be missing in order to explain HD 209458b's large size. The most likely source of energy available is not in the planet's spin or orbit, but in the intense radiation received from the parent star. We show that the radius of HD 209458b can be reproduced if a small fraction $(\sim 1 \%)$ of the stellar flux is transformed into kinetic energy in the planetary atmosphere and subsequently converted to thermal energy by dynamical processes at pressures of tens of bars.
\end{abstract}

Key words. stars: individual: 51 Peg, HD 209458 - planets and satellites: general - stars: planetary systems

\section{Introduction}

The detection of planetary-mass companions in small orbits around solar-type stars has been a major discovery of the past decade. To date, 73 extrasolar giant planets (with masses $M \sin i<13 M_{\mathrm{J}}, M_{\mathrm{J}}$ being the mass of Jupiter and $i$ the inclination of the system) have been detected by radial velocimetry. Fifteen of these $(21 \%)$ have distances less than $0.1 \mathrm{AU}$, and ten $(14 \%)$ have distances less than $0.06 \mathrm{AU}$ (see Marcy et al. 2000 and the discoverers' web pages). This is for example the case with the first extrasolar giant planet to have been discovered, $51 \mathrm{Peg}$ b (Mayor \& Queloz 1995). These close-in planets form a statistically distinct population: all planets with semi-major axis smaller than $0.06 \mathrm{AU}$ have near-circular orbits while the mean eccentricity of the global population is $\langle e\rangle \approx 0.27$. This is explained by the circularization by tides raised on the star by the planet (Marcy et al. 1997). One exception to this rule, HD $83443 \mathrm{~b}(e=0.079 \pm 0.033)$, can be attributed to the presence of another eccentric planet in the system (Mayor et al. 2001). As we shall see, the planets inside $\sim 0.1 \mathrm{AU}$ also have very specific properties due to the closeness to their star and the intense radiation they receive. For this reason, following astronomical conventions, we choose to name them after the first object of

Send offprint requests to: T. Guillot, e-mail: guillot@obs-nice.fr this class to have been discovered: "51 Peg b-like" planets, or in short "Pegasi planets".

Such planets provide an unprecendented opportunity to study how intense stellar irradiation affects the evolution and atmospheric circulation of a giant planet. Roughly $1 \%$ of stars surveyed so far bear Pegasi planets in orbit, suggesting that they are not a rare phenomenon. Their proximity to their stars increases the likelihood that they will transit their stars as viewed from Earth, allowing a precise determination of their radii. (The probability varies inversely with the planet's orbital radius, reaching $\sim 10 \%$ for a planet at $0.05 \mathrm{AU}$ around a solartype star.) One planet, HD 209458b, has already been observed to transit its star every 3.524 days (Charbonneau et al. 2000; Henry et al. 2000). The object's mass is $0.69 \pm 0.05 M_{\mathrm{J}}$, where $M_{\mathrm{J}}=1.89 \times 10^{27} \mathrm{~kg}$ is the mass of Jupiter. Hubble Space Telescope measurements of the transit (Brown et al. 2001) imply that the planet's radius is $96300 \pm 4000 \mathrm{~km}$. An analysis of the lightcurve combined with atmospheric models shows that this should correspond to a radius of $94430 \mathrm{~km}$ at the 1 bar level (Hubbard et al. 2001). This last estimate corresponds to $1.349 \bar{R}_{\mathrm{J}}$, where $\bar{R}_{\mathrm{J}} \equiv 70000 \mathrm{~km}$ is a characteristic radius of Jupiter. This large radius, in fair agreement with theoretical predictions (Guillot et al. 1996), shows unambiguously that HD 209458b is a gas giant.

We expect that the evolution of Pegasi planets depends more on the stellar irradiation than 
is the case with Jupiter. HD 209458b and other Pegasi planets differ qualitatively from Jupiter because the globally-averaged stellar flux they absorb is $\sim 10^{8} \mathrm{erg} \mathrm{cm}^{-2}\left(10^{5} \mathrm{~W} \mathrm{~m}^{-2}\right)$, which is $\sim 10^{4}$ times greater than the predicted intrinsic flux of $\sim 10^{4} \mathrm{erg} \mathrm{cm}^{-2}$. (In contrast, Jupiter's absorbed and intrinsic fluxes are the same within a factor of two.) Several evolution calculations of Pegasi planets have been published (Burrows et al. 2000a; Bodenheimer et al. 2001), but these papers disagree about whether HD 209458b's radius can be explained, and so far there has been no general discussion of how the irradiation affects the evolution. Our aim is to help fill this gap.

Here, we quantify how atmospheric processes affect the evolution of Pegasi planets such as HD 209458b. First (Sect. 2), we show that the evolution is sensitive to the assumed atmospheric temperatures. This sensitivity has not previously been documented, and quantifying it is important because the temperature profiles appropriate for specific planets remain uncertain (e.g., no atmospheric radiative transfer calculation for HD 209458b yet exists). Our works suggests that the discrepancy between the predictions of Burrows et al. (2000a) and Bodenheimer et al. (2001) can be largely explained by their different assumptions about atmospheric temperature.

Second, the effect of atmospheric dynamics on the evolution has to date been neglected. For example, current models assume the day-night temperature difference is zero, despite the fact that substantial day-night temperature variations are likely. In Sect. 3.1 we demonstrate how the evolution is modified when a day-night temperature difference is included. Furthermore, the intense stellar irradiation will lead to production of atmospheric kinetic energy, and transport of this energy into the interior could provide a substantial energy flux that would counteract the loss of energy that causes planetary contraction. In Sect. 3.2 we investigate this effect.

The research has major implications for HD 209458b. Early calculations implied that Pegasi planets contract slowly enough to explain HD 209458b's large radius (Guillot et al. 1996; Burrows et al. 2000a). But recent calculations of irradiated atmospheres suggest that the actual deep atmosphere is colder than assumed (Goukenleuque et al. 2000). When such realistic temperatures are adopted (our Sect. 2), the planet contracts too fast and the radius is $\sim 0.2-0.3 \bar{R}_{\mathrm{J}}$ too small. Bodenheimer et al. (2001) argued that tidal heating from circularization of the orbit would slow the contraction, leading to a larger radius, but this is a transient process that would end $\sim 10^{8}$ years after the planet's formation. Instead we argue that kinetic energy produced in the atmosphere is transported into the interior and dissipated (Sect. 3.2). We show that plausible downward energy fluxes can slow or even halt the planet's contraction, allowing HD 209458b's radius to be explained.

In a joint paper (Showman \& Guillot 2002, Paper II) we consider the atmospheric dynamics of these planets, with emphasis on how the atmospheres respond to stellar heating and gravitational tidal interactions, and on the observable consequences.

\section{Sensitivity of evolution to atmospheric temperature}

The upper boundary condition of evolution models consists of a relationship between the effective temperature and some deeper temperature (say that at 10 bars) to which the model's interior temperature profile is attached. Here we show that the evolution is sensitive to the assumed relationship (i.e., to the assumed atmospheric temperature structure).

Before we begin, we provide some definitions. We define the effective temperature of the irradiated planet as

$4 \pi R^{2} \sigma T_{\mathrm{eff}}^{4}=L_{\mathbf{\bullet}}+L_{\mathrm{int}}$,

where $R$ is the planet's radius, $T_{\text {eff }}$ is its effective temperature, $\sigma$ is the Stefan-Boltzmann constant, $L_{\mathbf{o}}$ is the part of the stellar luminosity absorbed by the planet and $L_{\text {int }}$ is the intrinsic luminosity of the planet due to its cooling and contraction (and possibly other processes such as radioactivity or thermonuclear reactions in the case of massive objects).

The temperature corresponding to the intrinsic planetary flux, called the "intrinsic" temperature $T_{\text {int }}$, is defined by

$4 \pi R^{2} \sigma T_{\text {int }}^{4}=L_{\text {int }}$.

Similarly, we define $T_{\mathbf{O}}$ from the absorbed stellar luminosity $L_{\mathbf{a}} . T_{\mathbf{\Phi}}$ is the effective temperature towards which the planet tends as it cools and $L_{\text {int }}$ diminishes (see e.g. Hubbard 1977). It is a function of the Bond albedo (i.e. the ratio of the luminosity directly reflected to the total luminosity intercepted by the planet). $T_{\mathbf{\Phi}}$ can be viewed as the effective temperature reached by the planet after it has lost its internal heat, and is hence sometimes noted $T_{\text {eq }}$ (e.g. Guillot et al. 1996; Saumon et al. 1996).

\subsection{Atmospheric boundary conditions}

We consider two evolution models of HD 209458b based on the parameters listed in Table 1; the two models differ only in their prescription for the atmospheric boundary condition.

Our first evolution sequence, dubbed the "hot" case, uses the standard boundary condition from Guillot et al. (1996) and Burrows et al. (2001a). These papers adopted an atmospheric structure of an isolated object with the expected effective temperature, which provides a fair fit to the evolution of Jupiter. The surface boundary condition consists of a relationship between the temperature at the 10 bar pressure level $T_{\text {isolated, the effective tempera- }}$ ture $T_{\text {eff }}$ and the gravity $g$ of an isolated planet/brown dwarf derived by several authors (see Marley et al. 1996; Burrows et al. 1997):

$T(P=10$ bar $)=T_{\text {isolated }}\left(T_{\text {eff }}, g\right)$. 
Table 1. Parameters used in evolution models.

\begin{tabular}{lll}
\hline \hline Parameter & Value & References/remarks \\
\hline Evolution model & CEPAM & Guillot \& Morel $(1995)$ \\
\hline Mass & $M=0.69 M_{\text {Jup }}$ & $\left(\right.$ MJup $\left._{\text {J }} \equiv 1.89 \times 10^{30} \mathrm{~g}\right)$ \\
Absorbed stellar heat & $T_{\mathbf{\Phi}}=1400 \mathrm{~K}$ & \\
Radius & $R=1.35 \bar{R}_{\mathrm{J}}$ & $\left(\bar{R}_{\mathrm{J}} \equiv 7 \times 10^{9} \mathrm{~cm}\right)$ \\
\hline EOS & "interpolated" & Saumon et al. (1995) \\
Helium mass mixing ratio & $Y=0.30$ & Higher than solar in order to mimic a solar abundance of \\
Rosseland opacities & - & heavy elements \\
Rotation & 0 & Alexander \& Ferguson (1994) incl. interstellar grains \\
Core mass & 0 & Neglected \\
Atmospheric boundary & - & Not considered \\
& & From Marley et al. (1996); Burrows et al. (1997) \\
\end{tabular}

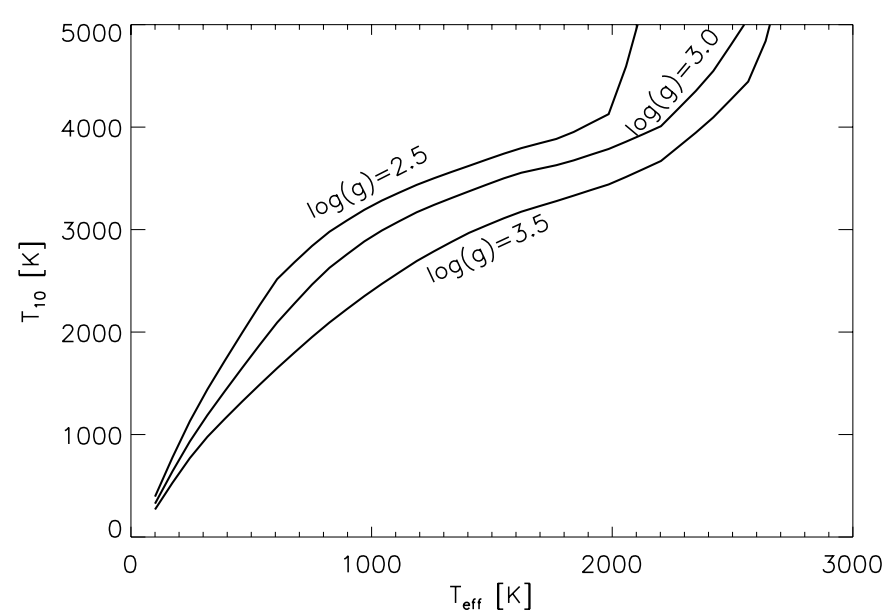

Fig. 1. Surface boundary condition (temperature at the 10 bar level) that has been used in several published evolution models, and which we dub the "hot" case, as a function of effective temperature for three different gravities: $\log (g)=2.5,3$ and 3.5. ( $g$ is in units of $\mathrm{cm} \mathrm{s}^{-2}$ ). (See Saumon et al. 1996 for a discussion.).

This approximation is exact in the limit when the stellar flux is entirely absorbed at the 10 bar level, or in a deep adiabatic (convective) region, as is the case for Jupiter (see Hubbard 1977 for a detailed discussion of the effect of insolation on Jupiter's evolution). Figure 1 shows the variation of $T_{\text {isolated }}$ with effective temperature and gravity, for values of interest in the case of Pegasi planets.

Unfortunately, the approximation becomes incorrect in the case of strongly irradiated planets because of the growth of a thick external radiative zone. Another boundary condition has therefore to be sought: either part of the stellar flux is able to penetrate to deeper levels $\left(P_{0}>10\right.$ bar $)$ and lead to a boundary condition defined by $T\left(P_{0}\right)>T_{\text {isolated }}$, or most of the stellar flux is absorbed at $P_{0}<10$ bar, yielding $T\left(P_{0}\right)<T_{\text {isolated. }}$ (This is due to the fact that in the radiative zone $\mathrm{d} T / \mathrm{d} P \propto F$, where $F$ is the flux to be transported.) It will be shown hereafter (see Sect. 3.3) that Eq. (3) is effectively an upper limit to the boundary temperature because continuum opacity sources only effectively limit the penetration of the stellar photons. Indeed, more detailed models of the atmospheres of Pegasi planets have shown that most of the starlight is absorbed at pressures less than 10 bar, and that Eq. (3) overestimates the atmospheric temperatures by as much as 300 to $1000 \mathrm{~K}$ (Seager \& Sasselov 1998, 2000; Goukenleuque et al. 2000; Barman et al. 2001).

Because these atmospheric models do not presently span the effective temperature and gravity range that is needed, and more importantly because they assume unrealistic intrinsic temperatures, we chose to construct an arbitrary boundary condition based on the results of the isolated case. For a given $T_{\text {eff }}$, the isolated case provides an upper bound to the "surface" temperature and by extension to the temperatures in the planetary interior. In order to have an approximate lower bound that agrees with atmospheric models of irradiated giant planets, we assume (i) a lower value of $P_{0}=3 \mathrm{bar}$, and (ii) that the temperature at that level is given by:

$T(P=3$ bar $)=T_{\text {isolated }}\left(T_{\text {eff }}, g\right)-1000 \mathrm{~K}$.

Evolution calculations made with this boundary condition are dubbed the "cold" case.

Note that we found a posteriori that the choice of $P_{0}$ is almost unconsequential for the evolution calculations. This is because the external radiative region quickly becomes almost isothermal (see Fig. 4 hereafter). However, the consequences of the cooler temperatures are profound, and as we shall see lead to a much faster evolution.

In this context, Bodenheimer et al. (2000, 2001) assume that the temperature at optical depth $2 / 3$ (corresponding in their model to a pressure of the order $\sim 1$ mbar) is equal to the effective temperature $T_{\text {eff }}$, an approximation that leads to an underestimation of the actual atmospheric temperatures. As a consequence, their 1 bar temperatures are of the order of $\sim 1400 \mathrm{~K}$, i.e. even lower than what is implied by Eq. (4). This would imply an extremely inefficient penetration of the stellar flux in the planetary atmosphere, in disagreement with detailed models of these atmospheres. We therefore prefer to use Eq. (4) as our "cold" boundary condition. 


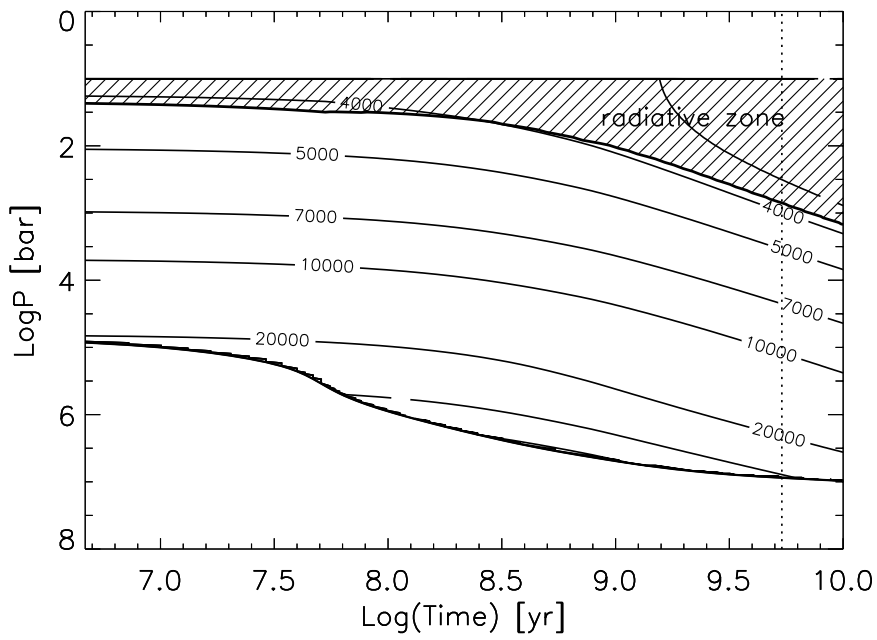

Fig. 2. Evolution of HD 209458b using the "hot" atmospheric boundary condition (Eq. (3)). The evolution of the central pressure with time is shown as the bottom thick line. The planet is convective except for an upper radiative zone indicated by a hashed area. Isotherms from 4000 to $20000 \mathrm{~K}$ are indicated. The isotherms not labelled correspond to 3500, 30000 and $40000 \mathrm{~K}$. The dashed line indicates the time necessary to con-

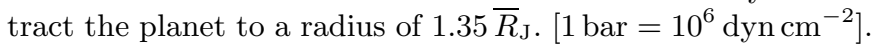

\subsection{Evolution models of HD $209458 b$}

The evolution of HD 209458b is calculated as described in Guillot et al. (1996), using the parameters given in Table 1. Because of the high stellar insolation, the contraction and cooling of the planet from a high entropy initial state is only possible through the build-up and growth of a radiative zone (Guillot et al. 1996).

The evolution of the interior of HD 209458b for the two cases is shown in Figs. 2 and 3. After a rapid contraction during which both the central pressure and temperature increase, the onset of degeneracy leads to a cooling of the interior as the planet continues to contract. The cooling of the interior proceeds despite the fact that the atmospheric temperatures remain nearly constant thanks to the growth of a radiative region in the planet's upper layers, as indicated by the dashed area.

In the case of the "hot" atmospheric boundary condition (Fig. 2), the measured radius $\left(1.35 \bar{R}_{\mathrm{J}}\right)$ is attained after $5.37 \mathrm{Ga}\left(5.37 \times 10^{9}\right.$ years $)$, which is compatible with the age of the G0 star HD 209458 (see Burrows et al. 2000a). The radiative zone then extends to about 730 bar, and the intrinsic luminosity is $7.7 \times 10^{24} \mathrm{erg} \mathrm{s}^{-1}$ (2.3 times that of Jupiter), which corresponds to an intrinsic temperature of $105 \mathrm{~K}$.

The "cold" atmospheric boundary condition (Fig. 3) yields a much faster evolution: the planet then shrinks to $1.35 \bar{R}_{\mathrm{J}}$ in only $0.18 \mathrm{Ga}$ (see also Fig. 6 hereafter). This is incompatible with the age derived for HD 209458b. The radiative/convective boundary in this model (at $0.18 \mathrm{Ga}$ ) is at $160 \mathrm{bar}$, due to the higher intrinsic luminosity equal to $1.9 \times 10^{26} \mathrm{erg} \mathrm{s}^{-1}$, equivalent to an intrinsic temperature of $234 \mathrm{~K}$.

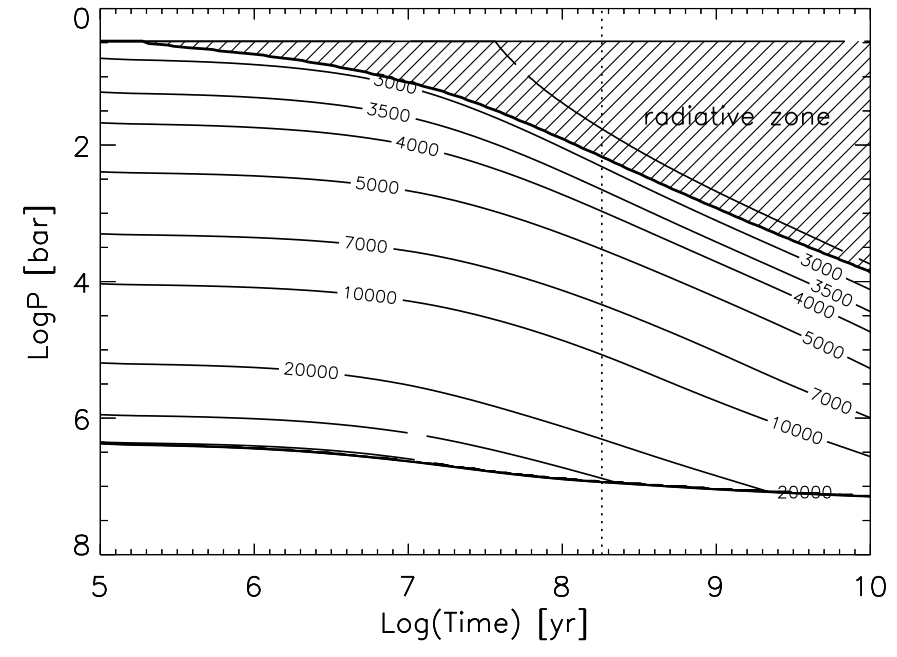

Fig. 3. Same as Fig. 2 but with the "cold" boundary condition given by Eq. (4). Unlabeled isotherms are for $T=2500,30000$ and $40000 \mathrm{~K}$.

The fact that the evolution is faster in the "cold" case may seem counterintuitive. It occurs because the intrinsic luminosity is proportional to the temperature gradient, not to the temperature itself. As shown by Fig. 4 hereafter, the temperature variation in the radiative region is more pronounced in the "cold" case than in the "hot" case. Basically, this is because the temperatures at deep levels are fixed by the condition on the radius, but that the surface temperatures are very different in the "cold" and "hot" cases.

Bodenheimer et al. (2001) obtain radii that slightly exceed those of our "cold" case, despite their lower atmospheric temperatures. However, this is probably due to their lower assumed value for the helium abundance $Y=0.24$, whereas we chose $Y=0.30$, a value representative of conditions in the solar nebula and that accounts for a solar proportion of heavy elements. In both cases, young ages are required to reproduce the measured planetary radius.

We therefore feel that because the "cold" atmospheric boundary condition is preferable to the "hot" boundary condition, there is a problem in explaining HD 209458b's radius. An absolute proof of this statement would require calculations of many different models using different assumptions, which we will not attempt in this paper. The conclusion should be relatively secure however, because several factors point towards a reduction of the planet's radius compared to what we have calculated: (i) the atmospheric temperatures could be even lower than in the cold case; (ii) the opacities used include the presence of abundant grains in the atmosphere and does not account for their gravitational settling; (iii) our choice of the equation of state tends to yield larger radii than would be the case using an equation of state that consistently models the molecular/metallic transition (see Saumon et al. 1995); (iv) the presence of a central core will tend to greatly reduce the planet's radius (Bodenheimer et al. 2001). 


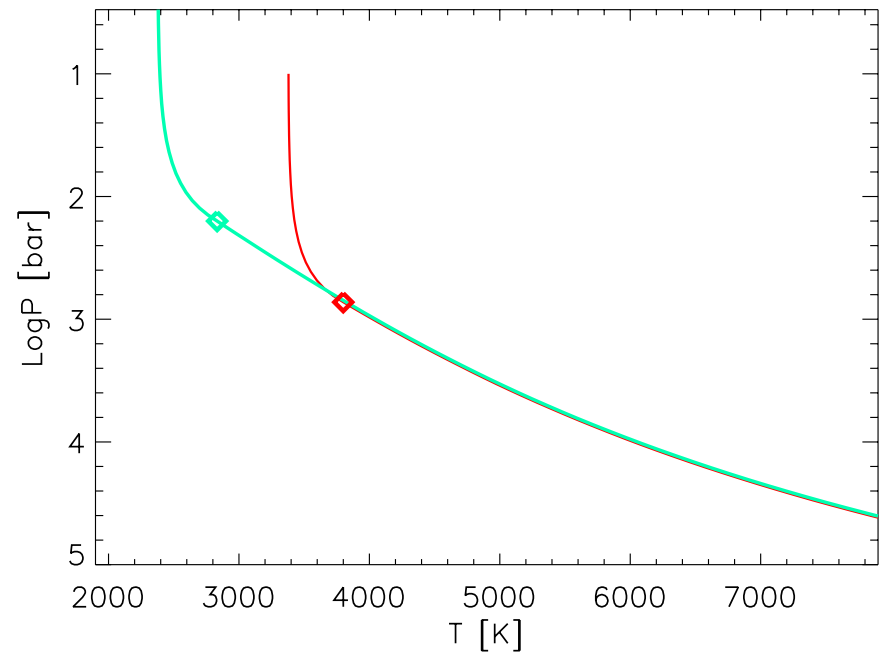

Fig. 4. Temperature profiles for the 5.37 Ga-old "hot" model (thin black line) and the 0.18 Ga-old "cold" model (thick grey line), i.e. when they match HD 209458b's measured radius. The diamonds indicate the radiative/convective boundary.

An additional source of energy then appears to be required. We note that the presence of a hydrogen/helium phase separation, like in Jupiter and Saturn (e.g. Stevenson \& Salpeter 1977; Guillot 1999), is not a valid alternative because of the high interior temperatures involved in the case of Pegasi planets (e.g. $20000 \mathrm{~K}$ at 1 Mbar).

An important aspect of the hot and cold models is shown in Fig. 4: apart from the outer radiative layers, the two models possess a very similar interior structure at the times $(5.37$ and $0.18 \mathrm{Ga}$ for the hot and cold cases, respectively) when they match HD 209458b's observed radius. This is easily understood by the fact that the radiative layer encompasses only a small fraction of the radius. Most of the contribution to the planetary radius is due to the convective interior. Fixing the radius is, for a given equation of state and composition, almost equivalent to fixing the temperature-pressure profile in the deep interior. HD 209458b can be thought of as a relatively wellconstrained convective core underlying a radiative envelope of uncertain mass and temperature.

\section{Evolution of Pegasi planets: “Non-standard" models}

\subsection{Implications of atmospheric day/night temperature variations}

We show in Paper II that the atmosphere should be significantly hotter on the dayside than the night side. Here we examine the consequences for the evolution models. To do so, we use a toy model that, while simple, elucidates the important physics. A full two-dimensional model that would allow us to calculate the evolution of Pegasi planets including latitudinal or longitudinal temperature variations will be left for future work.

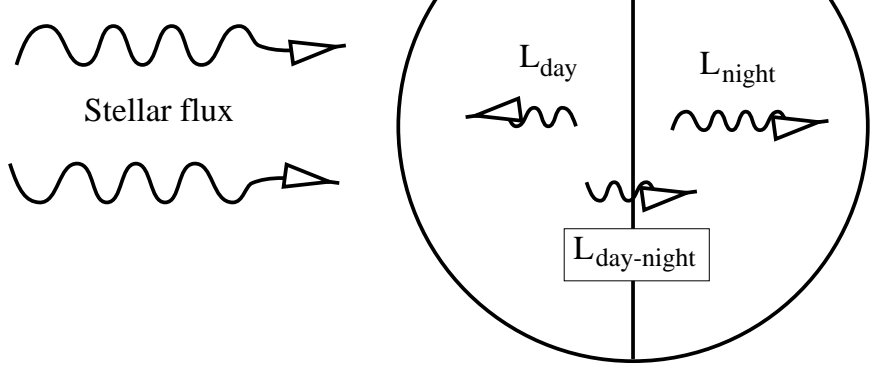

Fig. 5. Schematics of the day-night toy evolution model. The slow mixing of the interior leads to a non-radial heat flux $L_{\text {day-night }}$ from the day side to the night side. As a consequence, the intrinsic luminosity on the day side is smaller and that on the night side becomes larger.

Let us assume that the planet can be divided in two hemispheres (night and day) with two different effective temperatures such that $T_{\text {night }} \leq T_{\text {day }}$. When the absorbed stellar energy is fully redistributed by advection, $T_{\text {day }}=$ $T_{\text {night }}=T_{\mathbf{0}}$. In all cases, energy conservation implies that $2 T_{\mathbf{c}}^{4}=T_{\text {night }}^{4}+T_{\text {day }}^{4}$. Therefore, $T_{\text {night }}=0$ implies $T_{\text {day }}=$ $2^{1 / 4} T_{\mathbf{a}} ; T_{\mathbf{\Phi}}=1400 \mathrm{~K}$ and $T_{\text {day }}=1500 \mathrm{~K}$ yields $T_{\text {night }}=$ $1272 \mathrm{~K}$.

Let us do the following gedanken experiment, as illustrated by Fig. 5: we suppose that the two hemispheres cannot exchange energy, and let them evolve from the same initial state. After a given time interval $\Delta t$, the central entropy on the day side will have decreased by a smaller amount than on the night side. This is due to the fact that a higher atmospheric temperature is equivalent to a higher stellar flux, and leads to a slower evolution (see Hubbard 1977; Guillot et al. 1995). In consequence, the night side will have become internally colder, have a smaller radius and a larger central pressure than the day side.

The pressure differences caused by the differential cooling ensures an efficient mixing between the two hemispheres on a time scale of decades or less, i.e. much shorter than the evolution time scale.

We therefore include the effect of atmospheric temperature variations on the evolution in the following way: we calculate two evolution tracks of a planet with uniform temperatures $T_{\text {night }}$ and $T_{\text {day }}$, respectively. Using these evolution tracks and starting from an initial condition for which the two models have the same central entropy, we calculate the entropies of the two sides after a time interval $\delta t$. We then decrease the entropy of the day side and increase that of the night side so that both are equal to $\left(S_{\text {night }}+S_{\text {day }}\right) / 2$. The process is repeated for each time step. The cooling of the night side is therefore slowed by the mixing of material of slightly higher entropy from the day side, while the opposite is true for the day side.

The resulting evolution tracks are shown in Fig. 6. Not suprisingly, the cooling of an irradiated planet with inefficient heat redistribution in the atmosphere is faster 


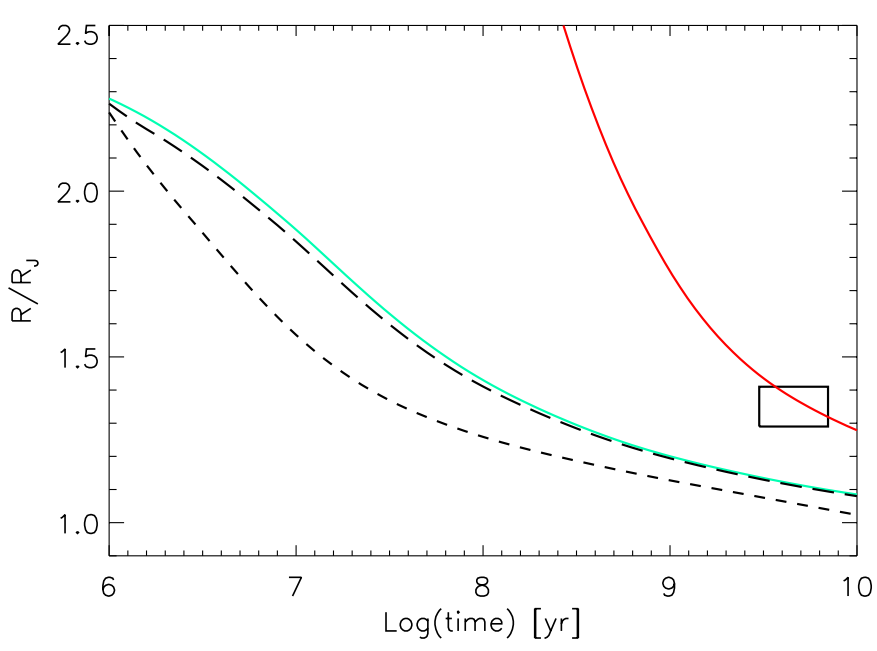

Fig. 6. Radius of HD 209458b (in units of the radius of Jupiter) versus time under different assumptions. The plain lines correspond to the "hot" and "cold" evolution cases shown in Figs. 2 (upper curve) and 3 (lower curve), respectively. The long dashed line is obtained in the "cold" case, when assuming that the radiative equilibrium effective temperature is $1500 \mathrm{~K}$ on the day side and $1272 \mathrm{~K}$ on the night side. The short dashed line is obtained when these effective temperatures are $1664 \mathrm{~K}$ and $0 \mathrm{~K}$, respectively ( $\sim$ no advection). The box indicates inferred radii and ages of HD 209458b.

than if the stellar heat is efficiently advected to the night side. This is mainly due to the fact that, with increasing $\Delta T_{\text {day-night }}, T_{\text {night }}$ decreases much more rapidly than $T_{\text {day }}$ increases, yielding a much faster cooling of the night side. However, for the temperature variation of $228 \mathrm{~K}$ shown in Fig. 6 (long dashed line), the effect is limited to a variation of $\sim 0.5 \%$ of the radius after $1 \mathrm{Ga}$ of evolution or more. The effect is of course more pronounced if no thermal energy advection occurs in the atmosphere $\left(T_{\text {night }}=0\right)$. In that case (short dashed line), the minimal radius is, for a given mass, composition and stellar insolation, up to $5 \%$ smaller than calculated in the uniform case.

Figure 7 shows that a non-uniform atmosphere has a substantial effect on the planet's intrinsic flux $(F=$ $\left.L / 4 \pi R^{2}\right)$. After $4.5 \mathrm{Ga}$, the flux of our planet with $T_{\text {night }}=$ $1272 \mathrm{~K}$ and $T_{\text {day }}=1500 \mathrm{~K}$ is 6310 and $2950 \mathrm{erg} \mathrm{cm}^{-2} \mathrm{~s}^{-1}$ on the night and day sides, respectively, which can be compared with the measured intrinsic fluxes of Jupiter, $5440 \mathrm{erg} \mathrm{cm}^{-2} \mathrm{~s}^{-1}$ and Saturn, $2010 \mathrm{erg} \mathrm{cm}^{-2} \mathrm{~s}^{-1}$ (Pearl \& Conrath 1991). This process is analogous to that proposed by Ingersoll \& Porco (1978) to explain the uniform temperatures of Jupiter. Stellar insolation tends to suppress the planet's intrinsic heat flux, and so the planetary heat preferentially escapes in regions where the insolation is minimal.

\subsection{Evolution with internal energy dissipation}

Current models predict that several-Ga-old Pegasi planets have intrinsic heat fluxes of $\sim 10^{4} \mathrm{erg} \mathrm{s}^{-1} \mathrm{~cm}^{-2}$, which is about $10^{4}$ times less than the total lumi-

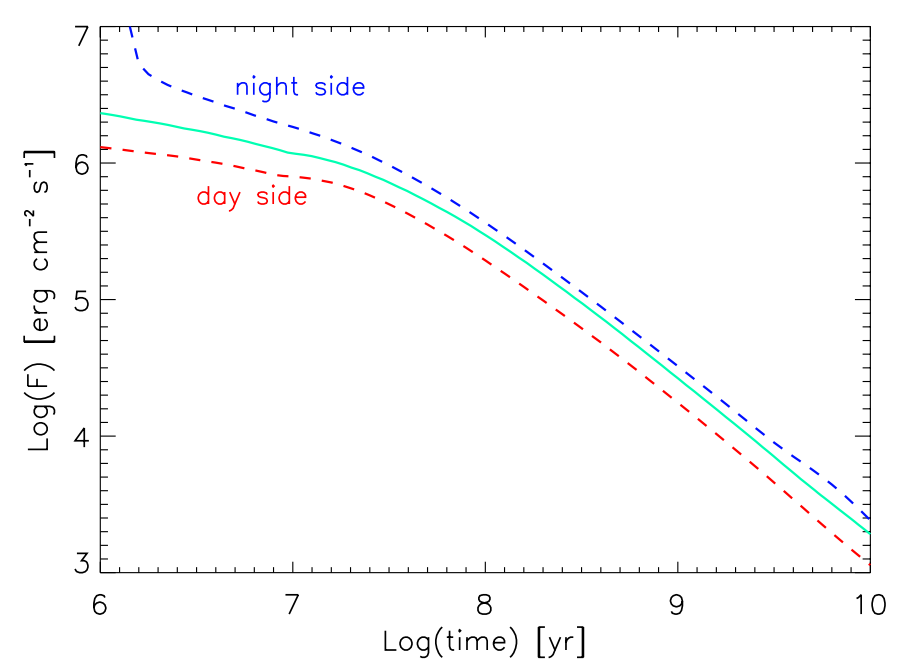

Fig. 7. Intrinsic planetary fluxes obtained as a function of time in the "cold" case with a well mixed atmosphere (plain line). When assuming that stellar irradiation is imperfectly redistributed over the planet's atmosphere, the flux on the night side $\left(T_{\text {night }}=1272 \mathrm{~K}\right)$ becomes larger than that on the day side $\left(T_{\text {day }}=1500 \mathrm{~K}\right)$.

nosity of $\sim 10^{8} \mathrm{erg} \mathrm{s}^{-1} \mathrm{~cm}^{-2}$ resulting from thermal balance with the stellar insolation. A fraction $\eta$ of the total luminosity will be converted into kinetic energy by the atmospheric pressure gradients. On Earth, the globally-averaged flux transported by the atmosphere is about $2.4 \times 10^{5} \mathrm{erg} \mathrm{s}^{-1} \mathrm{~cm}^{-2}\left(240 \mathrm{~W} \mathrm{~m}^{-2}\right)$, while about $2000 \mathrm{erg} \mathrm{s}^{-1} \mathrm{~cm}^{-2}$ is converted into large-scale atmospheric kinetic energy (Peixoto \& Oort 1992), leading to a value $\eta=0.01$. This energy production can be viewed as the work done by an atmospheric heat engine with an efficiency of $1 \%$. Preliminary simulations that we have conducted indicate that a similar ratio is relevant for Pegasi planets (Paper II). If so, the implied kinetic energy generation is $10^{2}$ times the intrinsic heat flux computed by current models. Inclusion of this energy could then lead to a first-order alteration in the behavior predicted in evolution models.

In steady state, the kinetic energy that is produced must be dissipated. On Earth, this dissipation mostly results from friction with the surface (Peixoto \& Oort 1992). For Pegasi planets, Kelvin-Helmholtz instabilities and breaking of gravity and planetary waves are more relevant. The key question is whether the energy is dissipated in the "weather" layer, where starlight is absorbed and radiation to space occurs, or in the deeper atmosphere. In the former case, the dissipation will provide only an order- $1 \%$ perturbation to the vertical profile of absorbed starlight and radiated thermal energy. In the latter case, it comprises a hundred-fold alteration in the interior energy budget. We therefore need to know (i) to what pressures can the energy be transported, and (ii) how deep must it be transported to cause a major effect on the interior?

As discussed in Paper II, mechanisms for transporting kinetic energy into the interior include Kelvin-Helmholtz instabilities, direct vertical advection, and waves. 
The dynamical coupling between atmospheric layers suggests that winds should develop throughout the radiative region even though the radiative cooling and heating occurs predominantly at pressures less than a few bars. The boundary between the radiative region and the convective interior (at 100-1000 bars depending on the model) is a likely location for dissipation, because Kelvin-Helmholtz instabilities and breaking of downward propagating waves can both happen there. Furthermore, application of the Taylor-Proudman theorem to the convective interior suggests that winds should develop throughout the convective interior even if the forcing occurs only near the top of the convective region. This increases the possibility of dissipation in the interior.

With the inclusion of an internal dissipative source, the energy equation becomes

$$
\frac{\partial L}{\partial m}=\dot{\epsilon}-T \frac{\partial S}{\partial t}
$$

where $m$ is the mass inside any given level, and $\dot{\epsilon}(m)$ is the energy dissipated per unit time per unit mass at that level.

The evolution of Pegasi planets including energy dissipation has been studied by Bodenheimer et al. (2001) in the context of the tidal circularization of the orbit of the planet. These authors focused on simulations where $\dot{\epsilon}$ was constant with $m$ (although they also performed some simulations with spatially-varying dissipation). The major difficulty is, as noted by the authors, the fact that the present eccentricities of extrasolar planets within $0.1 \mathrm{AU}$ of their star are small and that the detected Pegasi planets generally do not possess close massive planetary companions which would impose on them a forced eccentricity.

Instead, we argue that kinetic energy, generated from a portion of the absorbed stellar flux, is transported to the interior where it can be dissipated. Although the depth of such dissipation is unknown, the majority could be deposited within the radiative zone rather than throughout the interior. Due to the rapid rise of the Rosseland opacity with pressure and temperature, the effect of heating anywhere within the convective core is essentially equivalent to the case where it occurs entirely at the center (a result shown by Bodenheimer et al.). The question is whether even shallower heating - say that occurring at tens to hundreds of bars, where atmospheric kinetic-energy deposition is likely - can affect the evolution. Therefore, we here explore the influence of the dissipation's depth dependence and magnitude $\dot{E}=\int \dot{\epsilon} \mathrm{d} m$.

An ad hoc, but reasonable, assumption is that a fraction of up to $1 \%$ of the absorbed stellar flux is dissipated inside the planet. Quantitatively, we use $\dot{E}=$ $2.4 \times 10^{27} \mathrm{erg} \mathrm{s}^{-1}$. Relatively small values of $\dot{E}$ can affect the evolution, provided they are comparable or larger than the luminosity obtained without dissipation $L$ (note that $L \sim 10^{24}-10^{25} \mathrm{erg} \mathrm{s}^{-1}$ ) and affect the radiative gradient on a sufficiently extended region of the interior.

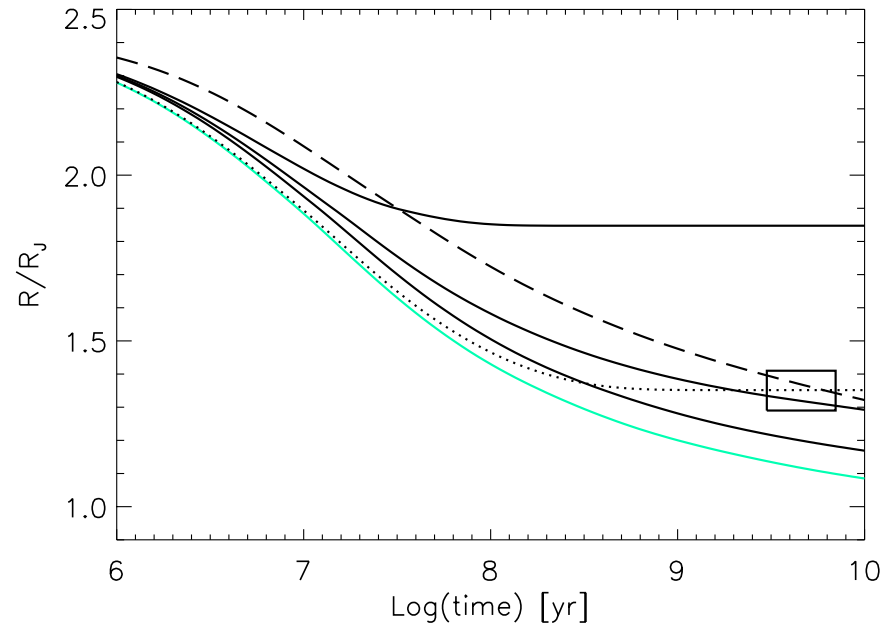

Fig. 8. Evolution tracks obtained in the "cold" case, showing the influence of dissipation. The bottom grey line corresponds to the case with no dissipation. The other solid lines have been calculated including the dissipation of $1 \%$ of the absorbed stellar flux $\left(2.4 \times 10^{27} \mathrm{erg} \mathrm{s}^{-1}\right)$, at various depths: from bottom to top, dissipation was supposed to occur in various mass shells: $\tilde{m}_{0}=10^{-5}, 2 \times 10^{-5}$, or at the center of planet, respectively. The dashed line corresponds to dissipation of $10 \%$ of the absorbed stellar flux $\left(2.4 \times 10^{28} \mathrm{erg} \mathrm{s}^{-1}\right)$ with $\tilde{m}_{0}=5 \times 10^{-6}$. The dotted evolution track is for dissipation of $\dot{E}=1.8 \times 10^{26} \mathrm{erg} \mathrm{s}^{-1}$ at the planet's center. In the first two cases, energy dissipation occurs mostly from the upper boundary to the nearly-isothermal region. The $\tilde{m}_{0}=5 \times 10^{-6}$, $10^{-5}$ and $2 \times 10^{-5}$ values of adimensional mass correspond to pressures of 5,11 , and 21 bars, respectively.

A first calculation assumes that energy is dissipated entirely at the center of the planet. In that case, as shown in Fig. 8 (uppermost solid line), an equilibrium with the star is reached after only $\sim 100 \mathrm{Ma}$, at which point the planet's radius is $1.87 \bar{R}_{\mathrm{J}}$ and its structure remains unchanged with time (as long as the star is also in equilibrium). This is very similar to the results obtained by Bodenheimer et al. (2001). Also in agreement with their results, we find that a calculation with the same $\dot{E}$, but with the dissipation evenly distributed throughout the interior (i.e., uniform $\dot{\epsilon}$ ) yields a curve similar to the upper solid line in Fig. 8.

In order to estimate the consequences of energy dissipation occurring closer to the planet's surface, we use the following arbitrary functional form:

$\dot{\epsilon}=\dot{\epsilon}_{0} \mathrm{e}^{-(1-\tilde{m}) / \tilde{m}_{0}}$,

where $\tilde{m}$ is the adimensional mass (0 at planet's center and 1 at its surface), $\tilde{m}_{0}$ is the mass fraction of the external regions over which most of the dissipation occurs, and $\dot{\epsilon}_{0}$ is chosen such that $\int \dot{\epsilon} \mathrm{d} m=\dot{E}=2.4 \times 10^{27} \mathrm{erg} \mathrm{s}^{-1}$. We will use values of $\tilde{m}_{0}$ equal to $5 \times 10^{-6}, 10^{-5}$, and $2 \times 10^{-5}$, which correspond to locations where the pressure is 5,11 , and 21 bars, respectively.

A choice of $\tilde{m}_{0}=10^{-5}$ (which implies dissipative heating distributed dominantly from the top boundary to 15 bar but with a tail of heating reaching $~ 100$ bar) yields 


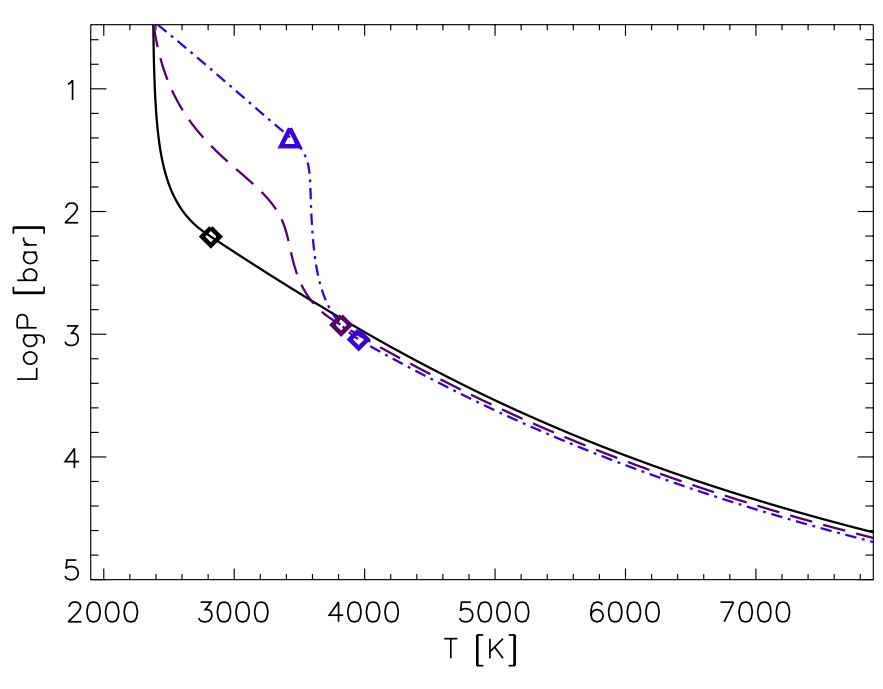

Fig. 9. Temperature-pressure profiles for models of HD 209458b (with $R=1.35 \bar{R}_{\mathrm{J}}$ ) calculated with the "cold" atmospheric boundary condition, and assuming various dissipation profiles: $\dot{E}=1.8 \times 10^{26} \mathrm{erg} \mathrm{s}^{-1}$ at the planet's center (solid line), $\dot{E}=2.4 \times 10^{27} \mathrm{erg} \mathrm{s}^{-1}$ ( $1 \%$ of the globally-averaged absorbed stellar heat flux) and $\tilde{m}_{0}=2 \times 10^{-5}$ (dashed line), $\dot{E}=2.4 \times 10^{28} \mathrm{erg} \mathrm{s}^{-1}$ and $\tilde{m}_{0}=5 \times 10^{-6}$ (dot-dashed line). The diamonds indicate the radiative/convective boundary. In the case of the dot-dashed models, the large dissipation in the external layers is responsible for a second convective zone pressures lower than 25 bars, as indicated by a triangle.

a radius which is, after a few billion years, about $10 \%$ larger than in the case with no dissipation (third solid line from the top in Fig. 8). This is, in the "cold" case, insufficient to reproduce the observed radius of HD 209458b. A slightly higher value of $\tilde{m}_{0}=2 \times 10^{-5}$ yields an evolution track which is in agreement with the measured radius, as shown in Fig. 8 (second solid line from the top). In that case, the value of $1-\tilde{m}=\tilde{m}_{0}$ corresponds, for the model with $1.35 \bar{R}_{\mathrm{J}}$ to a pressure level $P=28 \mathrm{bar}$ and $T=2800 \mathrm{~K}$. However, because of the form of Eq. (6), dissipation becomes negligible only around $1-\tilde{m} \approx 10 \tilde{m}_{0}$, i.e. $P=130$ bar and $T=3380 \mathrm{~K}$.

Two other evolution tracks have been calculated specifically to illustrate how HD 209458b's radius can be reproduced with different values of $\dot{E}$ and $\tilde{m}_{0}$. In the case of dissipation at the center, we were able to match an equilibrium radius of $1.35 \bar{R}_{\mathrm{J}}$ with $\dot{E}=1.8 \times 10^{26} \mathrm{erg} \mathrm{s}^{-1}$ (dotted line in Fig. 8), which is only $0.08 \%$ of the globalmean absorbed stellar flux. In the case of dissipation limited to a shallow layer $\left(\tilde{m}_{0}=5 \times 10^{-6}\right.$, corresponding to a pressure of 5 bars), we found that a relatively high value of $\dot{E}$ corresponding to $10 \%$ of the absorbed stellar flux was necessary for the planet to contract to its present radius in about $\sim 5 \mathrm{Ga}$ (dashed line in Fig. 8).

The temperature pressure profiles of three models calculated with the "cold" atmospheric boundary conditions but with different values of the dissipation factor, and such that their total radius is $1.35 \bar{R}_{\mathrm{J}}$, are compared in Fig. 9. The temperature profile of the model with dissipation at the center (solid line) is essentially indistinguishable from our reference "cold" model which included no dissipation but had a high intrinsic heat flux due to its young age. As dissipation is increased but at the same time limited to shallower outer layers, the temperature profile becomes more similar to the "hot" case shown in Fig. 4. In the case of the highest dissipation considered here but with a small $\tilde{m}_{0}=5 \times 10^{-6}$, an external (detached) convective region can form. Note that in this case $T_{\text {int }}=790 \mathrm{~K}$; atmospheric models calculated with these high intrinsic effective temperatures are also found to possess a deep convective zone (Barman et al. 2001).

\subsection{Penetration of the stellar flux into the deep atmosphere}

We have shown that a kinetic energy flux corresponding to a small fraction of the stellar flux can, if dissipated deep enough, significantly affect the planet's evolution. The result would be exactly the same if the stellar flux was radiatively transported to these deep levels. We argue that stellar heat cannot be deposited so deep, however.

As shown by Figs. 4 and 9 , the temperature profile of HD 209458b must cross the point defined by $P_{\mathrm{i}} \approx 1 \mathrm{kbar}$, $T_{\mathrm{i}} \approx 4000 \mathrm{~K}$, assuming that the planet is of solar composition (the addition of heavy elements tends to increase the temperature required at a given pressure, but doesn't otherwise alter the conclusions that follow). This point and the external boundary condition then define the intrinsic luminosity required to reproduce the measured radius, i.e.

$L_{\mathrm{int}} \approx \frac{64 \pi \sigma G M}{3} \frac{<T^{3}>}{<\kappa>}\left(\frac{\mathrm{d} T}{\mathrm{~d} p}\right)_{\mathrm{rad}}$,

where $\sigma$ is the Stefan-Boltzmann constant, $\langle T\rangle$ and $\langle\kappa\rangle$ are a mean temperature and opacity, respectively, and $(\mathrm{d} T / \mathrm{d} p)_{\mathrm{rad}}$ is the mean temperature lapse rate in the radiative zone.

In the "hot" case, the value of $L_{\text {int }}$ thus derived is small because the difference in temperature between the bottom of the radiative zone and the external boundary is small. This implies that HD 209458b needs to be relatively old to have such a low intrinsic luminosity. In the more realistic "cold" case, the planet either has to be uncomfortably young, or some additional heat has to be transported to these levels. This requires $\dot{\epsilon}>0$, but with the additional requirement that the temperature should be brought close to $\sim 3200 \mathrm{~K}$ at a pressure $P<P_{\mathrm{i}}$.

By definition of the optical depth $\tau_{\lambda}$, the proportion of stellar flux still remaining at a given level is equal to $\mathrm{e}^{-\tau_{\lambda}}$, where

$\tau_{\lambda}=\int_{z}^{\infty} \kappa_{\lambda} \rho \mathrm{d} z$

and $\kappa_{\lambda}$ is the monochromatic opacity, and $z$ is the altitude in the atmosphere. Equation (8) is strictly valid only in a one-dimensional approximation, but this is sufficient here. It is useful to approximate the integral by assuming a constant opacity and density in a slice of atmosphere of 
height equal to the pressure scale height. In that case, the pressure at which $99 \%$ of the stellar flux has been absorbed can be estimated by:

$$
P \lesssim 5 \frac{g}{\kappa_{\min }}
$$

where $\kappa_{\text {min }}$ corresponds to the minimum value of the opacity $\kappa_{\lambda}$.

We estimate from the previous section that a penetration of $1 \%$ of the stellar flux to $P \sim 100$ bar in the "cold" case allows the radius of HD 209458 b to be explained without any other energy dissipation. $99 \%$ of the flux of a $6000 \mathrm{~K}$ black body is emitted between 0.22 and $4.9 \mu \mathrm{m}$. The measured radius and mass implies that $g \approx$ $10^{3} \mathrm{~cm} \mathrm{~s}^{-2}$, therefore requiring $\kappa_{\min } \lesssim 5 \times 10^{-5} \mathrm{~cm}^{2} \mathrm{~g}^{-1}$.

This opacity is approximately the minimum expected for a pure hydrogen-helium mixture at $P \sim 1$ bar and $T \sim$ $1500 \mathrm{~K}$, at $\lambda \sim 1 \mu \mathrm{m}$ due to Rayleigh scattering by $\mathrm{H}_{2}$ and $\mathrm{H}_{2}-\mathrm{H}_{2}$ collision-induced absorption (see e.g. Lenzuni et al. 1991; Guillot et al. 1994). At temperatures above about $2000 \mathrm{~K}$, two very important sources of continuous opacity arise, led by the increasing number of free electrons: the free-free absoption of $\mathrm{H}_{2}^{-}$and the bound-free absorption of the $\mathrm{H}^{-}$ion. However the number of free electrons in a zero-metallicity gas remains low even at $3000 \mathrm{~K}$, and the low opacity minimum persists to temperatures exceeding $3000 \mathrm{~K}$, and pressures exceeding 10 bar. In this case a deep absorption of the stellar flux would then be likely.

However, in a mixture of solar-like composition, a large fraction of the electrons can be provided by alkali metals. Using electrons number densities obtained from Kurucz (1970) and Lodders (personal communication, 2001), we estimate the minimum continuous opacity to climb to $3 \times 10^{-3} \mathrm{~cm}^{2} \mathrm{~g}^{-1}$ at $2500 \mathrm{~K}$ and to $0.1 \mathrm{~cm}^{2} \mathrm{~g}^{-1}$ at $3500 \mathrm{~K}$, mostly due to $\mathrm{H}^{-}$absorption (John 1988). This alone prevents any relevant fraction of the stellar flux to reach levels at which the temperature is larger than $2500 \mathrm{~K}$.

Furthermore, a number of other opacity sources are expected to occur and even dominate the spectrum. Likely candidates are $\mathrm{K}$ and $\mathrm{Na}$ which are now known to contribute significantly to the atmospheric absorption of brown dwarfs with similar temperatures, at visible wavelengths (Burrows et al. 2000b). Similarly, TiO is expected to provide an even larger absorption at short wavelength where it appears in the deeper atmosphere. For example, Barman et al. (2001) find that $\tau_{1.2 \mu \mathrm{m}}=10$ is attained at pressures smaller than $\sim 6$ bar in the cloud-free atmosphere of Pegasi planets. Finally, clouds, if present, would cause an absorption of the stellar flux at even lower pressures.

It hence appears that only a zero-metallicity atmosphere would have a low-enough opacity to allow the stellar flux to penetrate to $P \sim 100$ bar. This is an unlikely possibility, the metallicity of HD 209458 being close to solar (Mazeh et al. 2000). One possibility remains however: that alkali metals and strong absorbers such as $\mathrm{TiO}$ are buried deep due to condensation effects on the night side (see Paper II), so that the atmosphere on the day side would be almost metal-free. It is not clear even in this case that the measured radius could be explained, because the lower overall opacities would increase the rate of cooling and hence contraction of the planet.

In all the cases considered here, it seems very difficult for the incoming stellar flux to penetrate down to levels where the temperature is large (more than $\sim 2500 \mathrm{~K}$ ). In order to reproduce HD 209458b's large radius, a temperature $\sim 4000 \mathrm{~K}$ at a pressure $P \sim 1$ kbar must be attained. Energy dissipation due to a transfer of kinetic energy hence appears as the most likely missing energy source.

\section{Conclusions}

We have shown that the evolution of Pegasi planets is mainly driven by processes occuring in their atmosphere and is consequently complex. The measurement of the radius of one of these objects, HD 209458b, has allowed us to probe some of these mechanisms in detail.

We demonstrated that radiative-equilibrium atmospheric models predicting temperatures above $\sim 2500 \mathrm{~K}$ at pressures $P \sim 10$ bar are unlikely given the rapid rise of the absorption with increasing temperature. Cooler temperatures are to be expected in the atmosphere and without other means than radiation to transport the incoming heat flux, HD 209458b's large radius cannot be reproduced unless the planet is much younger than is revealed by observations of its parent star.

We showed the atmospheric temperature variations to have a small effect on the planetary cooling, if limited to a few 100's K. The temperature variations lead to faster cooling of the planet compared to standard models, which assume the stellar heat to be evenly distributed onto the planet's atmosphere. This accentuates the problem of reproducing HD 209458b's radius.

Energy dissipation is however a very promising candidate to explain HD 209458b's missing heating source. Lubow et al. (1997) have shown that tidal synchronization of Pegasi planets could give rise to a large heat flux. But this mechanism is limited to the early evolution of the planet and should rapidly become negligible. Bodenheimer et al. (2001) argued that internal heating could be provided by tidal circularization of an eccentric orbit. This is similarly unlikely to occur in most Pegasi planets in the absence of a detected close, massive companion capable of exciting their eccentricity. The mechanism that we invoke is simply a downward transport of kinetic energy generated by the intense atmospheric heat engine. We showed that only $\sim 0.08 \%$ of the stellar flux has to be transported to the interior regions to explain the radius of HD 209458b. This fraction rises to $1 \%$ if heat dissipation occurs predominantly in the outer $2 \times 10^{-5}$ in mass (reaching down to $\sim 2 \times 10^{-4}$ ), or to $10 \%$ if it occurs predominantly in the outer $5 \times 10^{-6}$ (reaching down to $\sim 5 \times 10^{-5}$ ). Data for Earth show that $1 \%$ of the absorbed solar radiation is converted to kinetic energy and dissipated in the atmosphere, and $1 \%$ is plausible for Pegasi planets too. To alter the evolution, the energy need be deposited only a 
few scale heights below the altitude where it is created, lending plausibility to the idea.

The presence of energy dissipation may be quantified in the future when several Pegasi planets have been characterized. With several ground programs (STARE, VULCAN), accepted space missions (COROT, MONS, MOST) and proposed ones (KEPLER, EDDINGTON) aiming at detecting photometric transits of Pegasi planets, there is indeed a good chance that enough statistical information on the mass radius relationship of Pegasi planets can be gathered.

An unfortunate consequence of this study is that the possibility to determine the planets' compositions solely from their mass, radius and orbital characteristics seems to be postponed to a more distant future. On the other hand, we should rejoice over the perspective of better understanding of irradiated atmospheres and tidal dissipation. As usual, progress will mainly occur through observations and the direct characterization of Pegasi planets.

Acknowledgements. This work benefited from many interactions with M. S. Marley, and discussions over many years with members of the "Tucson group" (W. B. Hubbard, J. I. Lunine, A. Burrows). We also wish to thank P. Bodenheimer, R. Freedman, K. Lodders, and D. Saumon for a variety of useful contributions. This research was supported by the French Programme National de Planétologie, Institute of Theoretical Physics (NSF PH94-07194), and National Research Council of the United States. The numerical results described in this article are available from the following URL: http://www.obs-nice.fr/guillot/pegasi-planets.

\section{References}

Alexander, D. R., \& Ferguson, J. W. 1994, ApJ, 437, 879

Barman, T., Hauschildt, P. H., \& Allard, F. 2001, ApJ, 556, 885

Bodenheimer, P., Hubickyj, O., \& Lissauer, J. J. 2000, Icarus, 143, 2

Bodenheimer, P., Lin, D. N. C., \& Mardling, R. 2001, ApJ, 548,466

Brown, T. M., Charbonneau, D., Gilliland, R. L., Noyes, R. W., \& Burrows, A. 2001, ApJ, 552, 699

Burrows, A., Marley, M. S., Hubbard, W. B., et al. 1997, ApJ 491, 856

Burrows, A., Guillot, T., Hubbard, W. B., et al. 2000a, ApJ, 534, L97
Burrows, A., Marley, M. S., \& Sharp, C. M. 2000b, ApJ, 531, 438

Charbonneau, D., Brown, T. M., Latham, D. W., \& Mayor, M. 2000, ApJ, 529, L45

Goukenleuque, C., Bézard, B., Joguet, B., Lellouch, E., \& Freedman, R. 2000, Icarus, 143, 308

Guillot, T. 1999, Science, 286, 72

Guillot, T., Gautier, D., Chabrier, G., \& Mosser, B. 1994, Icarus, 112, 337

Guillot, T., Chabrier, G., Gautier, D., \& Morel, P. 1995, ApJ, 450, 463

Guillot, T., Burrows, A., Hubbard, W. B., Lunine, J. I., \& Saumon, D. 1996, ApJ, 459, L35

Guillot, T., \& Morel, P. 1995, A\&AS, 109, 109

Henry, G. W., Marcy, G. W., Butler, R. P., \& Vogt, S. S. 2000, ApJ, 529, L41

Hubbard, W. B. 1977, Icarus, 30, 305

Hubbard, W. B., Fortney, J. J., Lunine, J. I., et al. 2001, ApJ, submitted

Ingersoll, A. P., \& Porco, C. C. 1978, Icarus, 35, 27

John, T. L. 1988, A\&A, 193, 189

Kurucz, R. L., Smithsonian 1970, Obs. Spec. Rep., 309, 1

Lenzuni, P., Chernoff, D. F., \& Salpeter, E. E. 1991, ApJS, 76, 759

Lubow, S. H., Tout, C. A., \& Livio, M. 1997, ApJ, 484, 866

Marcy, G. W., Butler, R. P., Williams, E., et al. 1997, ApJ, 481,926

Marcy, G. W., Cochran, W. D., \& Mayor, M. 2000, Protostars and Planets IV, ed. V. Mannings, A. P. Boss, \& S. S. Russell, 1285

Marley, M. S., Saumon, D., Guillot, T., et al. 1996, Science, 272, 1919

Mayor, M., \& Queloz, D. 1995, Nature, 378, 355

Mayor, M., Naef, D., Pepe, F., et al. 2001, in Planetary Systems in the Universe: Observation, Formation and Evolution, IAU Symp., 202, ed. A. Penny, P. Artymowicz, A.-M. Lagrange, \& S. Russel, ASP Conf. Ser, in press

Mazeh, T., Naef, D., Torres, G., et al. 2000, ApJ, 532, L55

Pearl, J. C., \& Conrath, B. J. 1991, J. Geophys. Res., 96, 18921

Peixoto, J. P., \& Oort, A. H. 1992, Physics of Climate, American Institute of Physics, New York

Saumon, D., Chabrier, G., \& Van Horn, H. M. 1995, ApJS, 99, 713

Saumon, D., Hubbard, W. B., Burrows, A., et al. 1996, ApJ, 460, 993

Showman, A. P., \& Guillot, T. 2002, A\&A, 385, 166 (Paper II)

Seager, S., \& Sasselov, D. D. 1998, ApJ, 502, L157

Seager, S., \& Sasselov, D. D. 2000, ApJ, 537, 916

Stevenson, D. J., \& Salpeter, E. E. 1977, ApJS, 35, 239 